\title{
Discurso de inauguración de la XIX Reunión del Consejo Directivo de ALANAM* $^{*}$
}

\author{
ALEJANDRO GOIC G. ${ }^{1}$
}

\section{Keynote address to the $19^{\text {th }}$ Congress of ALANAM}

In his keynote address to the international seminar of the ALANAM (Association of Latin American National Academies of Medicine), held October 28-30, 2010, in Santiago, Chile, Dr. Alejandro Goic, President of the Chilean Academy of Medicine, discussed the state of health and of medical and health research in Latin American countries. He called attention to the fact that the National Academies of Medicine are learned and honorific institutions whose main function is to reflect on, and foster, medical practice, medical education, and public health. He noted that medical doctors bear a great individual and collective responsibility in the organization and management of sanitary services, but that health care as such concerns all citizens. Poverty is one of the most important factors conditioning the state of health in any society, particularly in developing countries. Because of the very nature of the medical profession, doctors are acquainted not only with the physical and mental ailments that afflict the poor, but also with their precarious housing, income, and labor conditions, as well as with how difficult it is for them to have access to good health care and education. He emphasized that health care is not only a technical, administrative and economic institution, but a moral one as well. When the economic considerations of the health care industry and its financial mechanisms prevail over the medical needs of the general population, a severe social problem arises to the extent that important segments of the population are denied access to health care, and preventable human suffering is left unattended. Society and governments have the responsibility of financing health services and ensure that the humanitarian ends of medicine are met in health care services and sanitary institutions. The superior aim in health care should always be to afford a humane, caring, and respectful relationship between health care professionals and all users of the health care system without exception.

(Rev Med Chile 2010; 138: 1553-1557). health.

Key words: Academies and Institutes; Health policy; Health transition; Public

Te arrulla el mar, te velan las montañas,

Te arde la frente y por los pies tiritas;

Con sus próvidas manos infinitas

Dios está removiendo tus entrañas.

E s la voz del poeta español Enrique DíezCanedo, una descripción imaginativa de los rasgos distintivos de la singular geografía de Chile y, talvez, una premonición de sus turbulen-
*Pronunciado en la Ceremonia Inaugural de la Reunión de ALANAM, en la sede del Instituto de Chile y sus Academias. Santiago de Chile, 28 de octubre, 2010.

1Presidente de ALANAM y de la Academia Chilena de Medicina. 
edificios patrimoniales; miles de puestos de trabajo se perdieron y, como consecuencia, se incrementaron los índices de pobreza. El daño estimado es de 30 mil millones de dólares.

Por cierto, la mayor desgracia fue la muerte de más de quinientas personas y, arrastrados por el mar, alrededor de un centenar de desaparecidos. La ayuda de los países, de nuestro y de otros continentes, fue pronta, generosa y eficaz. Con todo, Chile o "una loca geografía", al decir del escritor nacional Benjamín Subercaseaux, está hoy erguido, reconfortando sus dolores y restañando sus heridas, con renovada fe en su porvenir.

En memoria y homenaje de las víctimas de esta enorme tragedia que azotó a nuestro país, solicito a ustedes que nos pongamos de pie y guardemos un momento de silencio.

En esta especial ocasión, quisiera también expresar nuestro respeto y admiración a los valerosos mineros que fueran rescatados de las profundidades de una montaña en el desierto árido de Atacama. En contraste dramático con la muerte de cientos de personas inocentes que ocurre día a día en todo el mundo, víctimas de la guerra, el odio y la violencia, veo en este admirable esfuerzo solidario para salvar a 33 hombres atrapados sin salida, un signo de amor por la vida que reconforta el espíritu y ennoblece la condición humana.

I

Hoy estamos convocados para dar comienzo oficial a la XIX Reunión de la Asociación Latinoamericana de Academias Nacionales de Medicina (ALANAM). Participan las Academias de Argentina, Bolivia, Brasil, Colombia, Costa Rica, Chile, Ecuador, México, Paraguay, Perú, Uruguay y Venezuela, además de la Real Academia de España. Desde 1967, fecha de fundación de ALANAM, sus presidentes y delegados permanentes se han reunido cada dos años en los diferentes países del continente para debatir materias de su preocupación. En estos seminarios internacionales han sido escudriñados, entre otros, temas de tanta importancia como los sistemas de salud, pobreza, agua potable, atención primaria, adolescencia y drogas, gerontología, formación de los médicos y ética médica.

El temario de la presente Reunión contempla la Situación de Salud y de la Investigación en Medicina y Salud en Latinoamérica. Sirven de marco de referencia general para el análisis de estos asuntos, la Declaración de Río de Janeiro sobre Medio Ambiente de 1992, la Declaración del Milenio de las Naciones Unidas del año 2000, suscrito por 184 Jefes de Estado y de Gobierno; y los Objetivos de Desarrollo del Milenio del Programa de Naciones Unidas para el Desarrollo (PNUD). No es casual que, de los ocho objetivos mencionados en la Declaración del PNUD, tres se refieran a problemas específicamente médicos: reducir la mortalidad infantil, mejorar la salud materna, combatir el VIH/SIDA, el paludismo y otras enfermedades; en tanto que, otros cuatro, a factores condicionantes de la salud: erradicar la pobreza extrema y el hambre, lograr la enseñanza primaria universal, promover la igualdad entre los géneros y la autonomía de la mujer y garantizar la sostenibilidad del medio ambiente.

En la etapa preparatoria de esta reunión, hemos recabado la información y opiniones de todas las Academias sobre los temas a tratar, las que, en documentos consolidados, servirán de base para los debates. Durante el seminario, expondrán sobres estos asuntos expertos de la Organización Panamericana de la Salud y los representantes de las diversas Academias. Al término del encuentro, emitiremos una Declaración sobre Politicas de Salud para la Región, una visión consensuada sobre asuntos de alta relevancia para el bienestar y el progreso de nuestros pueblos.

En el programa, se ha incorporado la "Conferencia Magistral Dr. Gonzalo Esguerra Gómez”, en recuerdo y homenaje de este destacado médico colombiano, quien fuera un impulsor de la creación de ALANAM, a través de una exitosa gestión diplomática. La Conferencia: "Arte y Medicina", será dictada por el Miembro de Número de la Academia Chilena Dr. Juan Verdaguer Tarradela. Además, distinguiremos hoy a los ex Presidentes de ALANAM: Drs. Rolando Calderón Velasco, Académico de Perú; Zoilo Cuellar-Montoya, de Colombia; Rodrigo Fierro Benítez, Plutarco Naranjo Vargas, Mario Paredes Suárez, de Ecuador y Misael Uribe Esquivel, de México; y a los ex Secretarios Alternos: Drs. Zoilo Cuéllar Montoya, de Colombia; Roberto Medina Santillán, de México; Alvaro Moncayo Medina, de Colombia y Eduardo Prettel Zárate, de Perú; quienes, con gran dedicación, han contribuido desinteresada y decisivamente a promover los elevados fines de esta institución latinoamericana y su continuo progreso: 
Cumplo con agradecer a los patrocinadores de este encuentro: en particular al Ministerio de Salud, en la persona del Dr. Jaime Mañalich Muxi, Ministro de la Cartera, así como al Colegio Médico de Chile, entidad representativa del gremio médico, presidida por el Dr. Pablo Rodríguez Whipple, quienes con su generosa contribución económica han hecho posible la realización de este encuentro; al Sr. José Luis Cea Egaña, Presidente del Instituto de Chile, institución que cobija a las seis Academias Chilenas: de la Lengua, de la Historia, de Ciencias Sociales, Políticas y Morales, de Ciencias, de Medicina y de Artes, en cuya sede tiene lugar esta Ceremonia y se desarrollarán nuestras reuniones de trabajo; a la Organización Panamericana de la Salud, en la persona de su Representante en Chile Dr. Rubén Torres, la que nos concedió su invaluable auspicio y la participación de sus expertos; $y$, finalmente, a los miembros de la Academia Chilena que han colaborado activamente en la preparación de este evento, en particular a su Secretario, Dr. Rodolfo Armas Merino.

En nombre de ALANAM y de la Academia Chilena de Medicina, les doy la más cordial bienvenida, deseando que su estada en Santiago sea grata, así como exitosas las deliberaciones que han de emprender. Un saludo especial a las delegaciones de Argentina, Colombia y México, cuyas Repúblicas, al igual que la de Chile, están celebrando este año el bicentenario de su vida independiente.

\section{II}

Excúsenme que, tomando ventajas de esta ocasión, me permita hacer algunas consideraciones relativas a las Academias y al tema central de nuestra reunión: la salud.

Sabemos que el origen de la voz Akademeia se sitúa en la Grecia Antigua, para denominar un pequeño lugar cercano a Atenas donde enseñaba Platón. Luego, se utilizó con el sentido de una escuela o institución de educación superior hasta que, en el Renacimiento, se generalizara la palabra universidad en su sentido moderno. Aún hoy día, el término Academia se emplea en muchos países para designar algunas escuelas secundarias y corporaciones de educación superior, incluidas algunas que enseñan materias específicas e, incluso, varios oficios. Sin embargo, ya desde el Renacimiento, la palabra Academia se había aso- ciado a sociedades ilustradas, que no son escuelas en el sentido común de la palabra y no tienen obligaciones docentes o de investigación formales.

El origen de las Academias, así entendidas, se remonta a los siglos XIII y XIV, siendo, en la época, corporaciones dedicadas al cultivo de la filosofía, poesía, literatura o arqueología. Las primeras Academias científicas aparecen en Europa en los siglos XVI y XVII y, en el siglo XVIII, las Academias de Medicina, siendo las más antiguas la Académie Royal de Chirurgie creada en Paris en 1731, antecesora de la actual Académie Nationale de Médicine; en España, la Academia Médica Matritense fundada en 1734, antecesora de la Real Academia Nacional de Medicina; y, en Rusia, la Academia Médico-Militar establecida en 1799. En América latina, las Academias de Argentina, Colombia y Perú datan del siglo XIX y, las de otros países miembros, del siglo XX.

Si intentáramos una definición de las Academias de nuestro tiempo, diría que son corporaciones doctas y honoríficas, generalmente establecidas con autoridad pública, destinadas a la reflexión intelectual, al cultivo y promoción de la literatura, de las artes y ciencias, o de un arte o ciencia en particular, en nuestro caso, de la Medicina.

El propósito fundamental de nuestras Academias es común: el estudio y cultivo de todos los variados asuntos relativos a la medicina, la salud pública y la educación médica, con el fin de promover su progreso y preservar sus valores históricos y éticos.

Debemos reconocer que las actuales Academias son instituciones sui generis, silenciosas, lejanas para el gran público, desprovistas de poder; y para algunos, entes casi incomprensibles en una época de tonalidades utilitarias. De ellas se esperan juicios independientes y serenos y que sean fieles a su autonomía y altruismo. Los Académicos hacen su tarea intelectual graciosamente, sin otro estímulo que su amor por el saber y la búsqueda de la verdad y la belleza. Su fuerza es la del conocimiento y la inteligencia, la idoneidad, la experiencia y prestigio de sus miembros. Convengamos que estos atributos sociales son escasos y un antídoto para lo trivial e irrelevante. A mi parecer, las Academias son corporaciones indispensables para preservar la salud intelectual, el sentido humano y espiritual de nuestras sociedades y servir de estímulo para que la juventud aprecie el valor del estudio y el reconocimiento social a quienes se dedican a tareas 
nobles y desinteresadas. No menos importante, los académicos pueden ser mensajeros privilegiados para lograr un mejor conocimiento y comprensión mutua entre nuestras naciones y fomentar la concordia y la paz

\section{III}

Los médicos tenemos una gran responsabilidad individual y colectiva en la organización y funcionamiento de los servicios sanitarios. Sin embargo, la salud no es un tema que concierne sólo a los médicos, sino que a todos los ciudadanos, por su gran relevancia social, económica y política. Estamos concientes de que los problemas de salud son complejos y de no fácil solución pero, también sabemos, que la salud es un bien universalmente deseado. Extendida al plano colectivo, la atención de salud, como cualquier otro servicio, tiene un elevado costo para un país, el que debe ser asumido socialmente por algún mecanismo financiero que la haga factible. Del punto de vista económico, no parece haber acuerdo unánime sobre cuál es el mecanismo más eficiente de asignación de recursos al sector salud y no somos, primariamente, los médicos los llamados a dilucidarlo. Pero si podemos constatar que, cuando la organización de la atención de salud y sus mecanismos financieros privilegian los intereses económicos sobre los requerimientos médicos y de salud de las personas, se genera un grave problema social, que se traduce en inequidad en el acceso de la población a una atención médica oportuna y de calidad y, por lo tanto, en un sufrimiento humano colateral que es de suyo evitable.

Es sabido que los médicos tienen una particular sensibilidad por los problemas sociales. Esto no es de extrañar, en razón de que, como pocas otras profesiones, en nuestra práctica cotidiana nos relacionamos con todos los sectores de la sociedad y somos testigos de las aristas de la pobreza, que condiciona para millones de personas una vida muy difícil, no pocas veces triste y desesperanzada. Conocemos, no sólo de sus dolencias físicas y mentales, sino que, también, de sus precariedades: la insuficiencia de sus viviendas, la escasez de sus ingresos monetarios, su inestabilidad laboral, su difícil acceso a una salud y educación de calidad. Esta experiencia social y vital de la profesión médica con factores condicionantes de la salud, es lo que los académicos por su trayectoria y experiencia acumulada, y en la etapa madura de su vida profesional, pueden aportar a sus respectivos países. Sabias son, a mi parecer, las autoridades de los Gobiernos que escuchan y valoran la opinión de las Academias sobre materias que son de alto interés público, para resolverlas con mejor conocimiento de causa e independencia de juicio; además, un modo inteligente de superar la tensión histórica entre el conocimiento y el poder.

\section{IV}

Las dificultades objetivas que enfrenta el sector salud no deben ser obstáculo para expresar nuestros puntos de vista y aspiraciones en el marco de los ideales de la profesión médica y en beneficio de la equidad social. Lo importante es no confundir lo esencial con lo instrumental y tener claro que, el propósito último que nos debe mover a los médicos, es cuidar debidamente a las personas que sufren.

Es a la sociedad y sus gobiernos a los que corresponde generar los recursos y los espacios apropiados para que en el sistema de salud se revele el sentido humanitario de la medicina. Esto se traduce en la exigencia de justicia en el acceso al bien de la salud, en promoverla, en prevenir las enfermedades, los accidentes y violencias y en preservar el medio ambiente. Y, en lo relativo al cuidado de las personas, en una atención médica oportuna y de calidad, una infraestructura sanitaria concebida en función de las necesidades humanas de los pacientes, en la disponibilidad de tecnologías de uso clínico de probada eficacia y en condiciones laborales adecuadas para los profesionales, técnicos y auxiliares de la salud.

Debo subrayar que la organización de la atención de salud no es sólo un problema técnico, administrativo y económico, sino que también moral. De allí que los principios fundacionales de la medicina debieran tener expresión en el modo cómo la sociedad concibe y organiza la atención de salud, así como en el comportamiento médico en el contexto más amplio de la atención sanitaria.

Los valores ético-asistenciales que proclamaron nuestros padres hipocráticos: idoneidad, respeto por la vida, justicia, no discriminación, humanitarismo, debieran servir de fuente de inspiración para definir las bases éticas de un sistema de salud. 
Estos valores, en su proyección social, encuentran expresión en aquellas comunidades en que sus miembros están ligados por un interés y compromiso común de concebir e implementar un sistema sanitario que beneficie a todos.
Pero hay más: nuestra convicción es que el objetivo superior en salud es lograr que los servicios sanitarios hagan posible un modo social de relación interhumana en la que prevalezcan la acogida, la amabilidad y el respeto por las personas. 